\title{
Annals of Behavioral Medicine
}

\section{Reflective and Automatic Processes in Healthcare Professional Behavior: A Dual Process Model Tested across Multiple Behaviors}

Justin Presseau, Marie Johnston, Tarja Heponiemi, Marko Elovainio, Jill J Francis, Martin P Eccles, Nick Steen, Susan Hrisos, Elaine Stamp, Jeremy M Grimshaw, Gillian Hawthorne, Falko F Sniehotta

\section{CITATION}

Presseau, J., Johnston, M., Heponiemi, T., Elovainio, M., Francis, J.J., Eccles, M.P., Steen, N., Hrisos, S., Stamp, E., Grimshaw, J.M., Hawthorne, G., Sniehotta, F.F. (2014). Reflective and automatic processes in healthcare professional behavior: A dual process model tested across multiple behaviors. Annals of Behavioral Medicine, 48, 347-358. doi: 10.1007/s12160014-9609-8 
Reflective and Automatic Processes in Healthcare Professional Behavior: A Dual Process Model Tested across Multiple Behaviors

\author{
Justin Presseau \\ Newcastle University, UK \\ Marie Johnston \\ University of Aberdeen, UK \\ Tarja Heponiemi and Marko Elovainio \\ National Institute for Health and Welfare, Finland \\ Jill J Francis \\ City University London, UK \\ Martin P Eccles, Nick Steen, Susan Hrisos, Elaine Stamp \\ Newcastle University, UK \\ Jeremy M Grimshaw
}

Department of Medicine, University of Ottawa and Clinical Epidemiology Program, Ottawa

Hospital Research Institute, Canada

Gillian Hawthorne

The Newcastle upon Tyne Hospitals NHS Foundation Trust, UK

Falko F Sniehotta

Newcastle University, UK

Author Note 
Justin Presseau, Institute of Health and Society, Newcastle University, UK; Marie Johnston, College of Life Sciences and Medicine, University of Aberdeen, UK; Tarja Heponiemi and Marko Elovainio, National Institute for Health and Welfare, Finland; Martin P Eccles, Nick Steen, Susan Hrisos, Elaine Stamp, Institute of Health and Society, Newcastle University, UK; Jill J Francis, City University London, London, UK; Jeremy M Grimshaw, Department of Medicine, University of Ottawa, and Clinical Epidemiology Program, Ottawa Hospital Research Institute, Ontario, Canada; Gillian Hawthorne, The Newcastle upon Tyne Hospitals NHS Foundation Trust, UK; Falko F Sniehotta, Institute of Health and Society, Newcastle University, UK.

The research was supported by a grant from Diabetes UK. JMG holds a Canada Research Chair in Health Knowledge Transfer and Uptake. FFS is funded by Fuse, the UK Clinical Research Collaboration Centre for Translational Research in Public Health, a UKCRC Public Health Research Centre of Excellence. Funding for Fuse from the British Heart Foundation, Cancer Research UK, Economic and Social Research Council, Medical Research Council, and the National Institute for Health Research. The authors would like to thank the primary care staff involved for their participation and Mrs Margaret Hunter for her views throughout the project.

Correspondence concerning this article should be addressed to Justin Presseau, Institute of Health and Society, Newcastle University, Baddiley-Clark Building, Richardson Road, Newcastle Upon Tyne, NE2 4AX, United Kingdom. Email: justin.presseau@ @cl.ac.uk 


\begin{abstract}
Background: Clinicians' behaviors require deliberate decision-making in complex contexts and may involve both impulsive (automatic) and reflective (motivational, volitional) processes.
\end{abstract}

Purpose: Test a dual process model applied to clinician behaviors in their management of type 2 diabetes.

Methods: Design: Six nested prospective correlational studies. Questionnaires were sent to doctors and nurses in 99 UK primary care practices, measuring reflective (intention, action planning, coping planning) and impulsive (automaticity) predictors for six guidelinerecommended behaviors: blood pressure prescribing $(N=335)$, prescribing for glycemic control $(N=288)$, providing diabetes-related education $(N=346)$, providing weight advice $(N=417)$, providing self-management advice $(N=332)$ and examining feet $(N=218)$.

Results: Respondent retention was high. A dual process model was supported for prescribing behaviors, weight advice, and examining feet. A sequential reflective process was supported for blood pressure prescribing, self-management and weight advice, and diabetes-related education.

Conclusions: Reflective and impulsive processes predict behavior. Quality improvement interventions should consider both reflective and impulsive approaches to behavior change.

Keywords: Clinician behavior; dual process; diabetes; motivation; volition; automaticity 
Prevailing theories of health-related behavior conceive behavior as a function of conscious reflection and active decisions prior to enactment based on perceived utility, outcomes, risk, capability, social influence, and/or intention (1-3). Such models account for notable variability in a range of behaviors related to the health of individuals (4) and those who provide them with healthcare (5). However, much of human behavior is cued by the environment and enacted without active reflection and decision-making. An emerging literature advocates consideration of non-conscious influences on behavior alongside wellestablished reflective processes. The aim of the present study was to elucidate and test a dual process model of behavior that simultaneously accounts for motivational and volitional reflective processes operating in parallel with an impulsive, automatic process.

\section{Towards theoretical combination and clarification}

A variety of dual process models have been proposed each using different descriptors of each process (see Evans 2008 (6) for a review). For the purposes of this paper, we use the terminology proposed in Strack and Deutsch's (7) Reflective-Impulsive Model. Dual process models propose that behavior results from the interplay of two cognitive processes operating in parallel: a reflective and an impulsive process. The reflective process, exemplified by most social cognition models (e.g., the theory of planned behavior (1)), is the effortful process of which the individual is consciously aware and in control, enacting behavior based on conscious deliberation. While the reflective process is characteristically slow and requires cognitive capacity to make decisions, perform calculations and weigh options, it provides the capability to make complex decisions and creatively solve difficult tasks. Four decades of research in social and health psychology have demonstrated the importance of the reflective process in determining behavior with the concept of intentionality being a prototypical feature of reflection and decision making. 
Intention is a central motivational determinant of behavior. However, intentions are quickly forgotten (8) and less likely to affect behavior when not represented in working memory. Reflective self-regulation is effortful, drawing on limited cognitive resources; the more we control our behavior using such a process, the more likely we are to deplete our cognitive resources (9). Thus despite the importance of the reflective process, clear gaps between strong intention and subsequent action (10) have been identified, resulting in research into post-intentional volitional processes to better understand how motivated individuals enact their good intentions (11). For example, action planning when, where and how to engage in a behavior is a reflective strategy involving planning courses of action contingent on encountering the 'when' and the 'where', such that when they are encountered, behavior can follow with less need for decision making in the situation $(11,12)$. Functionally similar coping planning involves planning how to deal with challenges that may arise thereby promoting enactment despite anticipated obstacles $(11,13)$. The Reflective-Impulsive Model does not go beyond intention as the proximal behavioral determinant within the reflective process; volitional factors (e.g., planning) may provide an important theoretical and practical advance.

While the reflective process regulates the behaviors that we perceive as free and willful, not all behavior is directly or exclusively the consequence of conscious, active and considered decisions and intentions. Dual process models propose a second process - the impulsive process - which requires little cognitive capacity, operates quickly and automatically, requiring minimal effort and is the default process determining behavior. Clusters of cognitive associations (schemata) are formed over time and reflect learned predispositions to act in a given way, cued by external (environmental) or internal (e.g., affective) reactions which, when cued, lead to action. Given its default role, the impulsive process drives behavior unless there is a need and capacity for conscious decision-making(7). 
The theoretical description of the relationship between the two processes as defined in the Reflective-Impulsive Model and subsequent operationalizations (14) is one defined by a) the reflective process having the capacity to create impulsive processes through repeated reflective processes over time, whilst acknowledging b) the competition for activation of one or the other process at any given time. It is also plausible that for some behaviors the two processes may operate synergistically.

\section{Automaticity as an indicator of the impulsive process}

Operationalizing a dual process model involves balancing the feasibility and validity of assessing indicators of the impulsive process. Most existing approaches to assessing impulsive indicators are based on lab tasks with limited generalizability, assessing implicit affect and cognition using reaction times or external cues to prime the activation of assumed mental representations (15). While providing critical opportunities for experimentation, field usage poses a challenge. A multi-method approach across studies to assessing features of the impulsive process may provide the closest approximation to its behavioral impact.

Habit strength has been proposed as an indicator of the impulsive process $(16,17)$. Habits are behaviors with a history of cue-contingent enactment such that the cue, rather than a process of deliberation, determines behavior. The impulsive process is characterized by the rapid activation of cognitive associations outside of awareness; thus the degree of habitualness of a behavior can be used to operationalize the impact of the impulsive process on behavior. Habit strength assessment has predominantly involved the Self-Report Habit Index (18), which has a number of limitations (19). Nevertheless, a subset of items within the Self-Report Habit Index focusing on assessing the degree of automaticity in a particular behavior circumvents some of the conceptual and measurement issues noted in the literature (20). 
Clinician behaviors represent a novel context in which to test a dual process model. Clinician behaviors typically involve both a learned component and a need to make reasoned decisions with clear and important consequences for patients' health and well-being and thus both may contribute to understanding clinicians' behavior. Combining insights from dual process models with contemporary theorizing in motivational and volitional processes would account for learned behavior, clinical expertise in decision-making and the resource pressures which limit capacity for active deliberation. A number of studies demonstrate that clinicians tend to be motivated to perform behaviors that are recommended by clinical practice guidelines. What is not clear is whether volitional factors (action and coping planning) mediate the relationship between motivation and action in clinicians.

Care is improving for primary care-based diabetes management, but there remain clear gaps in quality of care (21-27). Given the prevalence of diabetes, a better understanding of the factors involved in providing high quality care would contribute insight into ways of improving care. It is plausible that reflective and automatic processes impact behaviors differently. For instance, behaviors such as prescribing have immediate health implications for the patient (if prescribed appropriately, inappropriately or indeed omitted) and thus reflective processes may outweigh impulsive processes for such behaviors particularly when having to add additional medication or when running out of prescribing options (28). Advising and examining may involve automaticity to a greater extent given their potential proclivity to become routinized although also involving reflective consideration of individual needs.

Hypothesis 1: Consistent with a sequential reflective process involving motivational and volitional processes, we hypothesized an indirect relationship between intention (motivation) and clinician behavior via action planning and coping planning (volition). 
Hypothesis 2: Consistent with a dual process approach to behavior, we hypothesized that an impulsive process would operate in parallel alongside the sequential reflective process. Specifically, we hypothesize that automaticity will predict unique and independent variance in behavior not accounted for by variables in the reflective process. The hypotheses were specified a priori through an internal process of study protocol elaboration including hypothesis generation and analysis specification and approval by the principle investigator and study team prior to data analysis.

Our aim was to test a sequential reflective process alongside a parallel impulsive process across six different clinician behaviors involved in the management of type 2 diabetes. We hypothesized that the pattern of results would vary depending on the type of behavior. Hypothesis $3 a$ : For prescribing behaviors, which require the use of an extensive knowledge base and have strong potential adverse consequences for patient health if performed incorrectly we expected the reflective process to override the impulsive process. Hypothesis $3 b$ : For advising and foot examination, we expected both impulsive and reflective processes to be predictive, given that advice and examination is largely routinized but requires a degree of tailoring to individual patients' needs.

\section{Overall Methods}

This study used a prospective design over 12 months with six nested studies to assess primary care general practitioners' and nurses' self-reported provision of six different guidelinerecommended behaviors related to their management of patients with type 2 diabetes. The study was embedded within a wider study of type 2 diabetes care (29). The behaviors were specified to represent a range of important yet challenging clinician behaviors: prescribing to reduce blood pressure to $140 / 80 \mathrm{mmHg}$ even following previous management; prescribing to reduce $\mathrm{HbA} 1 \mathrm{c}$ levels to $<8.0 \%$ despite maximum dosage on two hypoglycemic drugs; 
providing weight management advice to people with a BMI > 30 even following previous management; providing diabetes-related education; providing diabetes self-management advice; and examining circulation and sensation in feet. All participants provided signed informed consent. Ethics approval was granted by the Newcastle and North Tyneside 2 Research Ethics Committee (07/H0907/102).

Participants: Eight-hundred and forty-three primary care doctors and nurses in 99 general practices from across the United Kingdom were invited to complete measures at baseline and baseline respondents to complete self-reported behavior measures 12 months later. Of the 843 doctors and nurses invited, 678 (80.4\%) returned baseline questionnaires.

Materials and Procedure: Baseline materials consisted of questionnaires that included measures of intention, action planning, coping planning, and automaticity for each of the six behaviors. For each section of the questionnaire, doctors and nurses were asked to first indicate whether it was within their role to perform the behavior, and if so, to proceed with completing the items in the section. Questionnaire items are summarized below for one of the six behaviors, with the remainder available (21) and in the Electronic Supplementary Material; psychometric properties reported below are representative of the other behaviors.

\section{Exemplar Measures}

\section{Behavior 1: Prescribe to reduce blood pressure to $140 / 80 \mathrm{mmHg}$}

The target, action, context and time (30) of each behavior was provided at the top of the section: "The questions in this clinical area ask about your prescribing of additional antihypertensive drugs, both in general and over the next 12 months, for patients with type 2 diabetes whose blood pressure (BP) is $5 \mathrm{~mm} \mathrm{Hg}$ above a target of $140 \mathrm{~mm} \mathrm{Hg}$ Systolic BP or $80 \mathrm{~mm} \mathrm{Hg}$ Diastolic BP even following previous management.” Intention was assessed using three items (1-strongly disagree to 7-strongly agree; $\alpha=.93$ ) sharing the stem: "In my 
management of patients with diabetes whose BP is $5 \mathrm{~mm} \mathrm{Hg}$ above target ..." followed by e.g.,: "I intend to prescribe an additional antihypertensive drug". Action Planning was assessed using three items (1-strongly disagree to 7-strongly agree; $\alpha=.94$ ) with the common stem "I have a clear plan of..." followed by items assessing how, under what circumstances and when they will prescribe an additional antihypertensive drug. Coping Planning was assessed using nine items (1-strongly disagree to 7-strongly agree; $\alpha=.95$ ) sharing the stem "I have made a clear plan regarding prescribing an additional antihypertensive drug for patients whose BP is $5 \mathrm{~mm} \mathrm{Hg}$ above target if..." followed by: nine potential obstacles such as "The patient has COPD" and "The clinic is busy and I am running 20 minutes late".

Automaticity was assessed using a four-item subscale of the Self-Report Habit Index, the SelfReport Behavioral Automaticity Index (20) (1-strongly disagree to 7-strongly agree; $\alpha=.87$ ). All items shared a common stem to indicate the behavior and the cue to which the behavior was contingent: "Prescribing an additional antihypertensive drug for any patient whose BP is $5 \mathrm{~mm} \mathrm{Hg}$ above target is something" followed by: "I do automatically", "I do without thinking", "I do without having to consciously remember", "I start doing before I realize I'm doing it".

Twelve months later, baseline respondents were asked to self-report their prescribing behavior. To ensure correspondence with the predictors, the target, action, context, and time was again made clear as follows: the question asks about "you prescribing additional antihypertensive drugs, both in general and over the past 12 months, for patients with type 2 diabetes whose blood pressure (BP) is $5 \mathrm{~mm} \mathrm{Hg}$ above a target of $140 \mathrm{~mm} \mathrm{Hg} \mathrm{Systolic} \mathrm{BP} \mathrm{or}$ $80 \mathrm{~mm} \mathrm{Hg}$ Diastolic BP, even following previous management”. Clinicians were then asked to complete a single item assessing the behavior: "Over the past 12 months, given 10 patients with diabetes whose BP was $5 \mathrm{~mm} \mathrm{Hg}$ above target, for how many did you prescribe an additional antihypertensive drug?" (0-10 scale) 
Planned Analyses: We ran bootstrapped multiple mediation models to test the study hypotheses for each of six behaviors (31). We tested for simultaneous indirect associations between intention and behavior via both action planning and coping planning, as well as a direct relationship between automaticity and behavior in parallel. We also added job title as a dummy coded variable to control for variability attributable to differences between nurses (coded 0) and doctors (coded 1), and for experience (years since qualified). We repeated the same analysis for all six behaviors.

The present study used the same dataset as a previously published study (32) but addresses different hypotheses. The previous study aimed to test single theories (i.e., social cognitive theory, theory of planned behavior, learning theory, action and coping planning) separately from each other as a test of established theory. The present study differs by testing different hypotheses and involving formal mediation analyses (31) testing whether volitional constructs (action and coping planning) mediated the relationship between a motivational construct (intention) and behavior whilst considering a parallel impulsive construct (automaticity); these had not previously been combined and tested together. In addition, the present study operationalized automaticity using the 4-item Self-Report Behavioral Automaticity Index (20) whereas the previous study tested the 12-item self-report habit index (18) and a different 2-item habit measure, both of which were tested separately from the other reflective constructs. Whilst based on the same data, the two studies thus address different research questions.

\section{Results}

Prescribing to reduce blood pressure to 140/80 $\mathrm{mm} \mathrm{Hg}$ and Prescribing for glycemic control when $\mathrm{HbAlC}>8.0 \%$ 


\section{Response rates and descriptives}

Of those returning baseline questionnaires, 389 (348 doctors, 41 nurses) completed baseline measures for blood pressure prescribing and 332 (285 doctors, 47 nurses) completed baseline measures for prescribing for glycemic control. Respectively, 335 (86.1\%) and 288 (86.8\%) provided prescribing behavioral data at 12 months. As shown in Table 1 and using Cohen's effect size conventions (33), associations between reflective and impulsive processes and prescribing behaviors were medium and associations between the variables within each process were medium to large. Neither years qualified nor job title (nurse or general practitioner) were associated with prescribing for blood pressure, whilst job title was associated with prescribing for glycemic control.

\section{$<$ Table 1 here>}

The model (1000 bootstrapped models) accounted for $14 \%$ of the variability in prescribing for blood pressure (Figure 1) and prescribing for glycemic control (Figure 2).

For blood pressure prescribing, the sequential reflective model was supported, with intention showing both a direct relationship with behavior $(B=0.49, \mathrm{SE}=0.15, p<0.01)$ and an indirect relationship with behavior via Action Planning $(B=0.11, \mathrm{SE}=0.06,95 \% \mathrm{CI} 0.00$ to 0.24) but not Coping Planning ( $B=-0.02, \mathrm{SE}=0.03,95 \% \mathrm{CI}-0.10$ to 0.04 ). A parallel impulsive process was also supported, with automaticity accounting for independent variability $(B=0.32$, $\mathrm{SE}=0.12, p<0.01)$. Years qualified and job title were not significant predictors of blood pressure prescribing.

For prescribing for $\mathrm{HbA1c}$, the sequential reflective model was partially supported, with intention showing a direct relationship with behavior $(B=0.36, \mathrm{SE}=0.17, p=0.04)$ but no indirect relationship with behavior via Action Planning $(B=0.06, \mathrm{SE}=0.08,95 \% \mathrm{CI}-0.07$ to 
0.25 ) or Coping Planning ( $B=.03, \mathrm{SE}=0.06,95 \% \mathrm{CI}-0.09$ to 0.16$)$. Support was found for a parallel impulsive process, with automaticity also accounting for variability in prescribing $(B=0.25, \mathrm{SE}=0.11, p=0.02)$. Job title was a significant predictor $(B=-0.81, \mathrm{SE}=0.41, p=0.048)$, but years qualified was not.

Overall, for prescribing the findings support a dual process account of clinician behavior. In addition, the reflective process operated sequentially through motivational and volitional processes for blood pressure prescribing, whereas motivation was not mediated through volitional processes for prescribing for glycemic control.

<Figure 1 here>

$<$ Figure 2 here $>$

Providing advice about diabetes self-management, diabetes-related education, advice about weight management for those with a BMI>30, and examining feet Response rates and descriptives

Of those returning baseline questionnaires, 407 (257 doctors, 150 nurses) completed baseline measures for advice about self-management, 415 (255 doctors, 160 nurses) for providing diabetes-related education, 489 (326 doctors, 163 nurses) for weight management advice and 311 (181 doctors, 130 nurses) for examining feet. Twelve months later, 332 (81.6\%) provided behavioral data for advice about self-management, 346 (83.4\%) for diabetes general education, $417(85.3 \%)$ for weight management advice, and $218(70.1 \%)$ for examining feet. Table 2 presents bivariate associations between all variables within each advising and examining behavior. For each behavior, associations between reflective and impulsive processes and behavior were medium (large for foot examination) and associations between the variables within each process were medium to large. Years qualified and job title (nurse or 
general practitioner) were both associated with providing weight advice and examining feet, whereas only job title was associated with providing diabetes-related education and providing advice about diabetes self-management.

<Table 2 here>

\section{Model testing}

The model accounted for $24 \%$ of the variability in providing advice about diabetes selfmanagement, $28 \%$ of the variability in providing diabetes-related education, $23 \%$ of the variability in providing weight advice and $58 \%$ of the variability in foot examination.

The sequential reflective model was supported for all advice behaviors. For providing advice about diabetes self-management (Figure 3), intention had a direct relationship with behavior $(B=0.49, \mathrm{SE}=0.16, p<0.01)$ and an indirect relationship with behavior via Coping Planning $(B=0.13, \mathrm{SE}=0.06,95 \% \mathrm{CI} 0.02$ to 0.27$)$ but not Action Planning $(B=-0.10, \mathrm{SE}=0.11$, 95\%CI -0.30 to 0.12 ). For providing diabetes-related education (Figure 4), intention had a direct relationship with behavior $(B=0.38, \mathrm{SE}=0.17, p=0.03)$ and an indirect relationship with behavior via Action Planning $(B=0.36, \mathrm{SE}=0.13,95 \% \mathrm{CI} 0.11$ to 0.65$)$ but not Coping Planning ( $B=0.05, \mathrm{SE}=0.05,95 \% \mathrm{CI}-0.05$ to 0.16$)$. For providing weight advice (Figure 5), intention had an indirect relationship with behavior via Coping Planning $(B=0.08, \mathrm{SE}=0.04$, 95\%CI 0.01 to 0.16 ) but not Action Planning ( $B=-0.02, \mathrm{SE}=0.04,95 \% \mathrm{CI}-0.10$ to 0.05 ). For foot examination (Figure 6), the reflective model was supported but as opposed to the advice behaviors, intention showed a direct $(B=0.76, \mathrm{SE}=0.17, p<0.01)$ but not an indirect relationship with behavior via Coping Planning $(B=0.12, \mathrm{SE}=0.09,95 \% \mathrm{CI}-0.01$ to 0.32$)$ or Action Planning ( $B=-0.003, \mathrm{SE}=0.10,95 \% \mathrm{CI}-0.21$ to 0.18$)$.

A parallel impulsive process was supported for providing weight advice $(B=0.41$, $\mathrm{SE}=0.10, p<0.01)$ and examining feet $(B=0.48, \mathrm{SE}=0.13, p<0.01)$, but not for providing 
advice on diabetes self-management $(B=0.13, \mathrm{SE}=0.10, p=0.22)$ or providing diabetes education $(B=0.02, \mathrm{SE}=0.09, p=0.83)$.

Job title was a significant predictor of advice about diabetes self-management $(B=-$ 0.96, $\mathrm{SE}=0.26, p<0.01)$, of providing diabetes-related education $(B=-0.97, \mathrm{SE}=0.23, \mathrm{p}<0.01)$, of weight management advice $(B=-1.06, \mathrm{SE}=0.22, p<0.01)$ and of foot examination $(B=-1.19$, $\mathrm{SE}=0.35, p<0.01)$. However, years qualified was only a significant predictor for providing diabetes-related education $(B=-0.03, \mathrm{SE}=0.01, p=0.02)$ and providing weight advice $(B=0.03$, $\mathrm{SE}=0.01, p<0.01)$.

Overall, the findings support a dual process account for providing weight management advice and for examining feet, but not for providing self-management advice or providing diabetes-related education. The latter two behaviors instead show evidence of largely being driven through a sequential motivational and volitional reflective process.

$<$ Figure 3 here >

$<$ Figure 4 here >

$<$ Figure 5 here>

$<$ Figure 6 here>

\section{Discussion}

The present study aimed to test a dual process model of behavior across six different behaviors performed by health professionals in the context of type 2 diabetes management in primary care. We found evidence supporting a dual process model of behavior in four of the six behaviors. A reflective process accounted for variability in all behaviors, suggesting that motivational processes remain key direct and indirect predictors of clinician behavior.

Intention was a direct predictor of five of the six behaviors, though also operating on behavior 
indirectly via volitional constructs. Partially supporting Hypothesis 1, we showed that volitional processes helped to explain how intention is translated into behavior for four of the six behaviors, demonstrating for the first time to our knowledge that a sequential reflective model of behavior contributes to understanding clinician behavior. This is important, as it provides key targets for intervention to improve clinical practice and thus the care received by people with diabetes. Interventions can be designed which incorporate behavior change techniques (34) to help clinicians translate their motivation into action, which, given the often high level of intention reported by clinicians, provides a potentially fruitful new avenue of reducing quality gaps in the care provided to people with diabetes.

Support was also found for a parallel impulsive process, with four of the six behaviors showing evidence of a role for automaticity in explaining clinicians' behavior, thereby supporting Hypothesis 2. Although habit has been investigated and found to be predictive in health professionals before (35-37), this is to our knowledge the first time that automaticity has been tested in health professionals, and operationalized within a dual process model of behavior. Taken together, findings show that a dual process model of behavior was supported for four of the six behaviors investigated. This is an important finding for two reasons: theoretically, for complex behaviors with salient consequences for the self and others performed in stable contexts, both processes may be relevant. Practically, this also highlights that interventions focusing exclusively on reflective processes (e.g., educational meetings, guideline dissemination) may miss an important aspect of clinician (and other types of) behavior: the automatic features of its pursuit. Without addressing both reflective and impulsive features of behavior, interventions may be undermined by short term change reverting back to routine behavior due to the lack of consideration for the impulsive influences on behavior that maintain its pursuit. 
When both processes are predictive of on-going behaviors, it may be more effective to address both rather than to target only the reflective process (e.g., providing information, changing outcome expectations, setting goals) or the impulsive process (e.g., contingent prompts and rewards). An intervention focusing only on the reflective pathway risks being undermined by the underlying impulsive process and the existing learned behaviors that are triggered by it; whereas an intervention focusing only on the impulsive process does not account for the importance of active decision making. Both can be considered and in so doing could provide a powerful and theoretically robust means of promoting change across different areas of behavioral medicine.

The relative importance of either process seems behavior-dependent. Automaticity was predictive of four of the six behaviors, with only providing self-management advice and providing diabetes-related education not showing a role for the impulsive process. These findings were not anticipated in developing our hypotheses and are counter to Hypotheses $3 \mathrm{a}$ and $3 b$. Prescribing behaviors were both driven directly by intention and in parallel by automaticity, with action planning additionally mediating intention for blood pressure prescribing. Behaviors with a very specific patient-related cue (i.e., blood pressure and glycemic control targets not being met, presenting for annual review which involves examination of feet, and providing weight advice specifically to people with a BMI >30) showed a role for automaticity, whereas the two other behaviors involved cues more broadly (i.e., all patients with type 2 diabetes registered with the practice) did not. This has implications for designing interventions, as targeting the impulsive system may require a more constrained specification of patient and/or environmental characteristics that would cue behavior automatically. When predictive, the impulsive process was always predictive alongside the reflective process. This suggests that for clinicians' behaviors, when there is a 
component of automaticity, this operates in concert with a reflective process, which is consistent good clinical practice and strong patient-clinician relationships.

Four behaviors investigated showed that the relationship between intention and behavior operated indirectly through post-intentional processes of action or coping planning. However, for prescribing for HbAlc and examining feet, intention was a direct predictor of behavior without a role for post-intentional processes, suggesting that there may be boundary conditions to using volitional processes for understanding intention-behavior relationships.

Forming action and coping plans requires deliberate consideration of when, where, and how a behavior will be performed and anticipation of barriers and planned solutions to overcome them; a necessarily reflective process. However, forming such plans is also proposed as one of the means by which the reflective process can access the impulsive process (15) and repeated planning and enactment may result in automatic processes developing over the one year follow-up period. Given the observational nature of the present study it is not clear how far in the past those who formed plans actually did, and whether their resulting reports of automaticity are a consequence of such planning; as both sets of measures were assessed at the same time, it is not possible to robustly test this. However, whilst none of the behaviors operated completely automatically, two behaviors (prescribing for HbA1c and examining feet) did not show a role for action or coping planning in mediating the relationship between intention and behavior. It is therefore possible that for those two behaviors, any planning that has been conducted may have had the desired transfer into automaticity; equally, it is possible that any planning that was conducted was insufficient. A randomized controlled trial with dual process-based process evaluation would help to test such a mechanism in this population. 


\section{Testing a dual process model in health professional behavior}

Biomedical research and clinical trial evidence provide steady opportunities to improve healthcare. Given the reach of primary care, uptake of evidence into practice has important implications for improving health. However, a consistent finding is that such evidence is slow to reach the care provided to patients. Addressing such quality gaps involves understanding and changing the behaviors (e.g., providing advice, prescribing, examining, intervening) of healthcare professionals providing care. Behavioral theories typically used to predict health behaviors have been demonstrated to account for similar amounts of variability in healthcare professional behaviors $(5,32)$. Nevertheless, such models do not capture features of the impulsive process, which may be particularly relevant to the behavior of healthcare professionals. The present study therefore extends previous research in this population, which tested existing theories separately (21), by testing a combined sequential reflective process involving motivational and volitional processes alongside a parallel impulsive process using novel measures of automaticity, controlling for possible differences due to experience or job title. To our knowledge, this is the first such test reported.

Given the repeated performance of behavior in clinical contexts with consistent cues to action, it is not surprising that the impulsive process is particularly relevant for understanding health professional behavior. What is perhaps more surprising is the lack of consideration for automaticity in current theorizing about health professional behavior change (17). Many common techniques used to change professional behavior such as adding prompts and cues may directly engage the impulsive process (34). The present study further underscores healthcare professional behavior as key facet of behavioral medicine, highlighting the opportunities that behavioral medicine-based approaches can provide for testing and applying theory to understand and change health professional behavior. 


\section{Future research}

The reflective process can be used to create associative links and thus 'program' the impulsive process. Indeed behavior change techniques such as action and coping planning have as their premise that ex-situ decision making can create representative links in memory that can be activated in-situ automatically without the need for decision making (15). Future research could use prospective planning as a basis to investigate reflective and impulsive processes in existing behavior alongside a newly promoted behavior to investigate the potential conflict between the impulsive and reflective processes. In addition, evidence from the habit formation literature shows that typically, initially reasoned behaviors can become habitual through repeated performance (38-41). Techniques such as habit formation, habit reversal, and behavioral practice/rehearsal (34) would be good candidates for targeting the impulsive process alongside techniques such as action and coping planning. This is particularly relevant for health professional behaviors which in some cases may be repeated many times a day across different clinical consultations.

Past research investigating primarily physical activity, eating and travel behaviors in the general public have posited an interaction between intention and automaticity. Some evidence suggests that intention is moderated by habit, such that the relationship between intention and behavior decreases as the level of habit increases (16). However, the generalizability of such findings is limited by the assumption that the two processes operate in competition, which as the present study shows, is not necessarily the case. Furthermore, the reduction of the role of the reflective process as habit increases is not consistent with the tenets of a dual process model. The reflective and impulsive processes are posited to operate in parallel, with the impulsive process 'always on' and the reflective process having the capacity to override the impulsive process when needed and when capacity is available. It therefore seems that rather than asking whether the two processes interact, it may be more 
fruitful to focus on identifying the boundary conditions which determine when either of the two processes is activated. The present study demonstrated that a key boundary condition is the nature of the behavior itself, underscoring the strength of the multiple behavior designs which allowed us to show that the impulsive process is overridden completely for two behaviors (self-management advice and providing diabetes-related education). Additional boundary conditions could be sought, which may include both states (14) and traits (42).

\section{Strengths and Limitations}

This study appears to be the first to model motivational and volitional processes of a sequential reflective process alongside features of an impulsive process in a sample of healthcare professionals. The study distinguishes itself by testing the model in multiple clinician behaviors in the same sample and by high response rates.

A recognized limitation of the Self-Report Habit Index is its inclusion of items of past behavior which draw on the same information as a frequency-based self-reported measure of behavior $(19,20,43)$. To address this, we instead used the Self-Report Behavioral Automaticity Index which omits items of frequency and focuses specifically on automaticity. In addition, we addressed a limitation of existing self-reported measure of automaticity by specifying the cue for which the behavior is contingent, providing a measure of automaticity with arguably greater construct validity that highlights cue contingencies. Although the measure of automaticity is self-reported and thus not a direct measure of the impulsive process, individuals are often aware of the consequences of their automatic behavior and can often recall their lack of awareness; this may be particularly true of healthcare professional behavior. Automaticity based on the Self-Report Behavioral Automaticity Index is one possible operationalization of the impulsive process, for which we have demonstrated predictive validity in this study. There is a need for additional sources of validation of the 
measure and more generally for development of measures of the impulsive process for use in applied health contexts.

Finally, while self-reported behavior may be limited by recall bias, our measure is strengthened by a long follow-up period. Future research should strive to use other objective measures of clinicians' behavior while recognizing their limitations. Clinicians' management of Type 2 diabetes in primary care is often team-based, posing a challenge in sourcing objective behavioral data attributable to individual clinicians, as routinely recorded outcomes are often only available at the team level. Such measures assume that the behavior of an individual is equivalent to the behavior of the entire team, which is likely to overestimate the behavior of a given individual and omits the variability between individuals as everyone from a team would be attributed the same behavioral score. While alternative measures of behavior could be used, such as coded video observations of individual clinical encounters or examining individual patient records, neither were feasible given the national scope of the present study and both have their own potential biases. We opted for self-reported behavior to ensure individually-linked behavior, maximizing the precision of the self-report using a clearly specified target, action, context and time (TACT, 30), and maximizing the TACTcorrespondence between measures of behavior to its predictors. A systematic review of social cognition models tested in health professionals highlighted that objective measures of behavior tended to have low TACT-correspondence with the theoretical predictors of the behavior (5). The validity of the self-report measure of behavior used in the present study is strengthened by the evidence of predictive validity in the supported hypotheses. Nevertheless, we acknowledge the inherent limitation of self-report measures of behavior and we recommend that future methodological work place increased attention on developing feasible, individually-attributable and valid measures of clinicians' behavior. 


\section{Conclusion}

Impulsive automatic processes operate in parallel with reflective motivational and volitional processes to predict primary care clinician behaviors. Quality improvement interventions should consider both reflective and impulsive approaches to behavior change.

Conflict of Interest Statement: Justin Presseau, Marie Johnston, Tarja Heponiemi, Marko Elovainio, Jill J Francis, Martin P Eccles, Nick Steen, Susan Hrisos, Elaine Stamp, Jeremy M Grimshaw, Gillian Hawthorne, and Falko F Sniehotta declare that they have no conflict of interest. All procedures, including the informed consent process, were conducted in accordance with the ethical standards of the responsible committee on human experimentation (institutional and national) and with the Helsinki Declaration of 1975, as revised in 2000. 


\section{References}

1. Ajzen I: The theory of planned behavior. Organizational behavior and human decision processes. 1991, 50:179-211.

2. Bandura A: Social foundations of thought and action: A social cognitive theory. Upper Saddle River, NJ: Prentice-Hall, 1986.

3. Schwarzer R: Modeling health behavior change: How to predict and modify the adoption and maintenance of health behaviors. Applied Psychology. 2008, 57:1-29.

4. McEachan RRC, Conner M, Taylor N, Lawton RJ: Prospective prediction of healthrelated behaviors with the Theory of Planned Behavior: A meta-analysis. Health Psychology Review. 2011, 5:97-144.

5. Godin G, Bélanger-Gravel A, Eccles MP, Grimshaw JM: Healthcare professionals' intentions and behaviours: A systematic review of studies based on social cognitive theories. Implementation Science. 2008, 3:36.

6. Evans JSBT: Dual-processing accounts of reasoning, judgment, and social cognition. Annual Review of Psychology. 2008, 59:255-278.

7. Strack F, Deutsch R: Reflective and Impulsive Determinants of Social Behavior. Personality and Social Psychology Review. 2004, 8:220-247.

8. Einstein GO, McDaniel MA, Williford CL, Pagan JL, Dismukes RK: Forgetting of intentions in demanding situations is rapid. Journal of Experimental Psychology: Applied. 2003, 9:147-162.

9. Baumeister RF, Bratslavsky E, Muraven M, Tice DM: Ego depletion: Is the active self a limited resource? Journal of Personality and Social Psychology. 1998, 74:1252-1265.

10. Orbell S, Sheeran P: "Inclined abstainers": A problem for predicting health-related behaviour. British Journal of Social Psychology. 1998, 37:151-165. 
11. Sniehotta FF: Towards a theory of intentional behaviour change: Plans, planning and self-regulation. British Journal of Health Psychology. 2009, 14:261-273.

12. Gollwitzer PM: Implementation intentions: Strong effects of simple plans. American Psychologist. 1999, 54:493-503.

13. Kwasnicka D, White M, Presseau J, Sniehotta FF: Does planning how to cope with anticipated barriers facilitate health-related behaviour change? A systematic review. Health Psychology Review. in press.

14. Hofmann W, Friese M, Wiers RW: Impulsive versus reflective influences on health behavior: a theoretical framework and empirical review. Health Psychology Review. 2008, 2:111-137.

15. Sheeran P, Gollwitzer PM, Bargh JA: Nonconscious processes and health. Health Psychology. in press.

16. Gardner B, de Bruijn G-J, Lally P: A systematic review and meta-analysis of applications of the self-report habit index to nutrition and physical activity behaviours. Annals of Behavioral Medicine. 2011, 42:174-187.

17. Nilsen P, Roback K, Broström A, Ellström P-E: Creatures of habit: accounting for the role of habit in implementation research on clinical behaviour change. Implementation Science. 2012, 7:53.

18. Verplanken B, Orbell S: Reflections on past behavior: A self-report index of habit strength. Journal of Applied Social Psychology. 2003, 33:1313-1330.

19. Sniehotta FF, Presseau J: The habitual use of the self-report habit index. Annals of Behavioral Medicine. 2012, 43:139-140.

20. Gardner B, Abraham C, Lally P, de Bruijn G-J: Towards parsimony in habit measurement: Testing the convergent and predictive validity of an automaticity subscale of 
the Self-Report Habit Index. International Journal of Behavioral Nutrition and Physical Activity. 2012, 9:102.

21. Eccles MP, Hrisos S, Francis JJ, et al.: Instrument development, data collection and characteristics of practices, staff and measures in the Improving Quality of Care in Diabetes (iQuaD) Study. Implementation Science. 2011, 6:61.

22. McFarlane SI, Jacober SJ, Winer N, et al.: Control of cardiovascular risk factors in patients with diabetes and hypertension at urban academic medical centers. Diabetes Care. 2002, 25:718-723.

23. Braga M, Casanova A, Teoh $\mathrm{H}$, et al.: Treatment gaps in the management of cardiovascular risk factors in patients with type 2 diabetes in Canada. Can J Cardiology. 2010, 26:297-302.

24. Hawthorne G, Hrisos S, Stamp E, et al.: Diabetes care provision in UK primary care practices. PLOS ONE. 2012, 7:e41562.

25. Khunti K, Ganguli S, Baker R, Lowry A: Features of primary care associated with variations in process and outcome of care of people with diabetes. British Journal of General Practice. 2001, 51:356-360.

26. Williams DR, Baxter HS, Airey CM, Alis S: Diabetes UK funded surveys of the structural provision of primary care diabetes services in the U. Diabetic Medicine. 2002, 19 Suppl 2:21-26.

27. Millett C, Car J, Eldred D, Khunti K, Mainous AG: Diabetes prevalence, process of care and outcomes in relation to practice size, caseload and deprivation: national crosssectional study in primary care. Journal of the Royal Society of Medicine. 2007, 100:275-283. 28. Verplanken B: Beyond frequency: Habit as mental construct. British Journal of Social Psychology. 2006, 45:639-656. 
29. Eccles MP, Hawthorne G, Johnston M, et al.: Improving the delivery of care for patients with diabetes through understanding optimised team work and organisation in primary care. Implementation Science. 2009, 4:22.

30. Fishbein M: Attitude and the prediction of behavior. In M. Fishbein (ed), Readings in attitude theory and measurement. New York: Wiley, 1967.

31. Hayes AF: Introduction to mediation, moderation, and conditional process analysis: A regression-based approach. New York: The Guilford Press, 2013.

32. Presseau J, Johnston M, Francis JJ, et al.: Theory-based predictors of multiple clinician behaviors in the management of diabetes. Journal of Behavioral Medicine. in press. 33. Cohen J: A power primer. Psychological Bulletin. 1992, 112:155-159.

34. Michie S, Richardson M, Johnston M, et al.: The behavior change technique taxonomy (v1) of 93 hierarchically clustered techniques: Building an international consensus for the reporting of behavior change interventions. Annals of Behavioral Medicine. in press.

35. Eccles MP, Grimshaw JM, Johnston M, et al.: Applying psychological theories to evidence-based clinical practice: Identifying factors predictive of managing upper respiratory tract infections without antibiotics. Implementation Science. 2007, 2:26.

36. Bonetti D, Johnston M, Clarkson JE, et al.: Applying psychological theories to evidence-based clinical practice: identifying factors predictive of placing preventive fissure sealants. Implementation Science. 2010, 5:25.

37. Grimshaw JM, Eccles MP, Steen N, et al.: Applying psychological theories to evidence-based clinical practice: identifying factors predictive of lumbar spine x-ray for low back pain in UK primary care practice. Implementation Science. 2011, 6:55.

38. Judah G, Gardner B, Aunger R: Forming a flossing habit: an exploratory study of the psychological determinants of habit formation. British Journal of Health Psychology. in press. 
39. Lally P, van Jaarsveld CHM, Potts HWW, Wardle J: How are habits formed:

Modelling habit formation in the real world. European Journal of Social Psychology. 2010, 40:998-1009.

40. Lally P, Wardle J, Gardner B: Experiences of habit formation: A qualitative study. Psychology, Health \& Medicine. 2011, 16:484-489.

41. Lally P, Gardner B: Promoting habit formation. Health Psychology Review. 2013, 7:S137-S158.

42. Sladek RM, Phillips PA, Bond MJ: Implementation science: a role for parallel dual processing models of reasoning? Implementation Science. 2006, 1:12.

43. Gardner B, Abraham C, Lally P, de Bruijn G-J: The Habitual Use of the Self-report Habit Index: A Reply. Annals of Behavioral Medicine. 2012, 43:141-142. 
Table 1

Descriptive statistics and correlations between reflective and impulsive processes for both prescribing clinician behaviors

Prescribing to reduce blood pressure to $140 / 80 \mathrm{mmHg}(\mathrm{N}=335)$

\begin{tabular}{lcccccc}
\hline & 1 & 2 & 3 & 4 & 5 & 6 \\
\hline 1. Behavior & $6.70(2.44)$ & & & & & \\
2. Intention & $0.33^{* *}$ & $5.51(1.04)$ & & & & \\
3. Action Planning & $0.24^{* *}$ & $0.43^{* *}$ & $5.94(0.83)$ & & & \\
4. Coping Planning & $0.18^{* *}$ & $0.39^{* *}$ & $0.45^{* *}$ & $4.66(1.19)$ & & \\
5. Automaticity & $0.30^{* *}$ & $0.52^{* *}$ & $0.28^{* *}$ & $0.49^{* *}$ & $3.98(1.31)$ & $22.25(8.40)$ \\
6. Years qualified & -0.05 & -0.04 & -0.10 & -0.04 & -0.10 & $-0.11^{*}$ \\
7. Job title & 0.04 & 0.11 & 0.00 & 0.00 & $0.12^{*}$ & $91.0 \% \mathrm{GPs}$ \\
\hline
\end{tabular}

\begin{tabular}{|c|c|c|c|c|c|c|c|}
\hline \multicolumn{8}{|c|}{ Prescribing to reduce $\mathrm{HbA1c}$ levels to $<8.0 \%$ (N=288) } \\
\hline & 1 & 2 & 3 & 4 & 5 & 6 & 7 \\
\hline 1. Behavior & $7.18(2.36)$ & & & & & & \\
\hline 2. Intention & $0.29 * *$ & $5.57(0.95)$ & & & & & \\
\hline 3. Action Planning & $0.27 * *$ & $0.51 * *$ & $5.67(1.04)$ & & & & \\
\hline 4. Coping Planning & $0.26 * *$ & $0.49 * *$ & $0.65 * *$ & $4.82(1.28)$ & & & \\
\hline 5. Automaticity & $0.29 * *$ & $0.50 * *$ & $0.42 * *$ & $0.51 * *$ & $4.01(1.47)$ & & \\
\hline 6. Years qualified & 0.07 & 0.06 & 0.08 & 0.08 & 0.02 & $22.59(8.24)$ & \\
\hline 7. Job title & $-0.18 * *$ & $-0.13 *$ & $-0.28 * *$ & $-0.19 * *$ & -0.08 & $-0.15^{*}$ & $87 \%$ GPs \\
\hline
\end{tabular}

$* * \mathrm{p}<.01 ; * \mathrm{p}<.05$. Note. Means (SD) presented along the diagonal. GPs $=$ general practitioners. 
Table 2

Descriptive statistics and correlations between reflective and impulsive processes for advising and foot examination clinician behaviors

Providing diabetes self-management advice $(N=332)$

\begin{tabular}{|c|c|c|c|c|c|c|c|}
\hline & 1 & 2 & 3 & 4 & 5 & 6 & 7 \\
\hline 1. Behavior & $7.98(2.28)$ & & & & & & \\
\hline 2. Intention & $0.41 * *$ & $5.82(1.11)$ & & & & & \\
\hline 3. Action Planning & $0.30 * *$ & $0.72 * *$ & $5.51(1.13)$ & & & & \\
\hline 4. Coping Planning & $0.37 * *$ & $0.61 * *$ & $0.61 * *$ & $4.76(1.35)$ & & & \\
\hline 5. Automaticity & $0.36 * *$ & $0.64 * *$ & $0.52 * *$ & $0.58 * *$ & $4.98(1.48)$ & & \\
\hline 6. Years qualified & 0.01 & $0.18 * *$ & $0.17 * *$ & $0.13^{*}$ & 0.07 & $22.66(8.22)$ & \\
\hline 7. Job title & $-0.35 * *$ & $-0.41 * *$ & $-0.37 * *$ & $-0.34 * *$ & $-0.37 * *$ & $-0.13 * *$ & $63 \%$ GPs \\
\hline \multicolumn{8}{|c|}{ Providing diabetes-related education $(N=346)$} \\
\hline & 1 & 2 & 3 & 4 & 5 & 6 & 7 \\
\hline 1. Behavior & $8.23(2.24)$ & & & & & & \\
\hline 2. Intention & $0.43 * *$ & $6.02(0.93)$ & & & & & \\
\hline 3. Action Planning & $0.45 * *$ & $0.70 * *$ & $5.66(1.11)$ & & & & \\
\hline 4. Coping Planning & $0.34 * *$ & $0.57 * *$ & $0.60 * *$ & $4.56(1.22)$ & & & \\
\hline 5. Automaticity & $0.33 * *$ & $0.62 * *$ & $0.51 * *$ & $0.53 * *$ & $4.98(1.48)$ & & \\
\hline 6. Years qualified & -0.03 & 0.10 & $0.12 *$ & 0.08 & 0.05 & $22.82(8.33)$ & \\
\hline 7. Job title & $-0.34 * *$ & $-0.36 * *$ & $-0.29 * *$ & $-0.25 * *$ & $-0.35 * *$ & $-0.16^{* *}$ & $61 \%$ GPs \\
\hline \multicolumn{8}{|c|}{ Providing weight management advice to people with a BMI > $30(N=417)$} \\
\hline & 1 & 2 & 3 & 4 & 5 & 6 & 7 \\
\hline 1. Behavior & $8.07(2.18)$ & & & & & & \\
\hline 2. Intention & $0.27 * *$ & $6.09(0.84)$ & & & & & \\
\hline 3. Action Planning & $0.12 *$ & $0.38 * *$ & $5.89(0.93)$ & & & & \\
\hline 4. Coping Planning & $0.27 * *$ & $0.44 * *$ & $0.32 * *$ & $4.44(1.26)$ & & & \\
\hline
\end{tabular}




\begin{tabular}{|c|c|c|c|c|c|c|c|}
\hline 5. Automaticity & $0.38 * *$ & $0.55 * *$ & $0.27 * *$ & $0.48 * *$ & $4.81(1.28)$ & & \\
\hline 6. Years qualified & $0.18^{* *}$ & 0.06 & -0.02 & 0.02 & 0.04 & $22.30(8.39)$ & \\
\hline 7. Job title & $-0.34 * *$ & $-0.21 * *$ & $-0.14 * *$ & $-0.13 *$ & $-0.30 * *$ & $-0.16^{* *}$ & $68 \%$ GPs \\
\hline \multicolumn{8}{|c|}{ Examining feet $(N=218)$} \\
\hline & 1 & 2 & 3 & 4 & 5 & 6 & 7 \\
\hline 1. Behavior & $7.63(3.05)$ & & & & & & \\
\hline 2. Intention & $0.69 * *$ & $6.00(1.30)$ & & & & & \\
\hline 3. Action Planning & $0.47 * *$ & $0.59 * *$ & $6.35(0.81)$ & & & & \\
\hline 4. Coping Planning & $0.56 * *$ & $0.61 * *$ & $0.64 * *$ & $5.77(1.31)$ & & & \\
\hline 5. Automaticity & $0.66 * *$ & $0.69 * *$ & $0.42 * *$ & $0.56 * *$ & $4.71(1.32)$ & & \\
\hline 6. Years qualified & $0.14^{*}$ & $0.16^{*}$ & 0.12 & $0.17^{*}$ & 0.10 & $23.28(8.65)$ & \\
\hline 7. Job title & $-0.58 * *$ & $-0.56 * *$ & $-0.43 * *$ & $-0.46 * *$ & $-0.55 * *$ & $-0.16^{*}$ & $51 \%$ GPs \\
\hline
\end{tabular}

$* * \mathrm{p}<.01 ; * \mathrm{p}<.05$. Note. Means (SD) presented along the diagonal. GPs $=$ general practitioners 


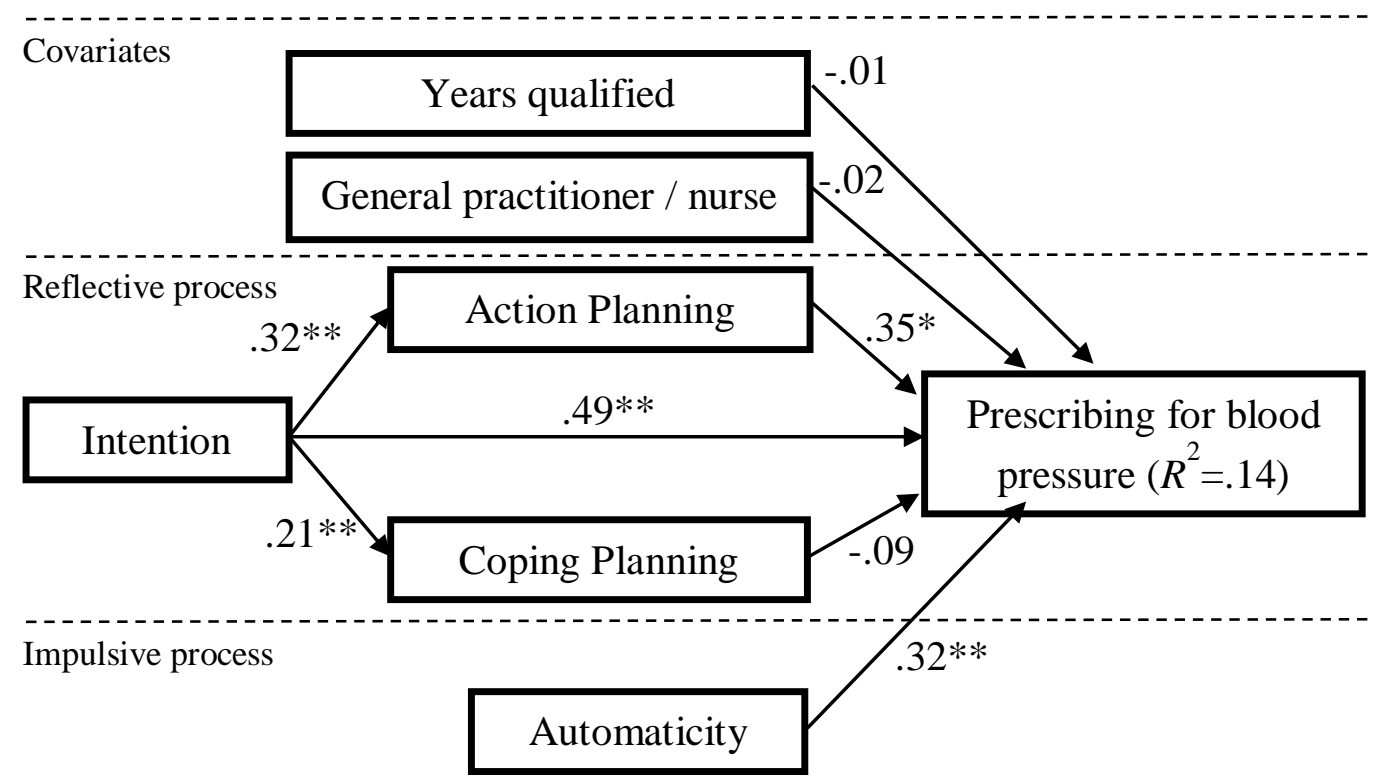

Figure 1. Prescribing for blood pressure: Bootstrapped multiple mediation model simultaneously testing the sequential relationship between intention and behavior via action and coping planning, alongside automaticity, controlling for differences between general practitioners and nurses and years qualified. 


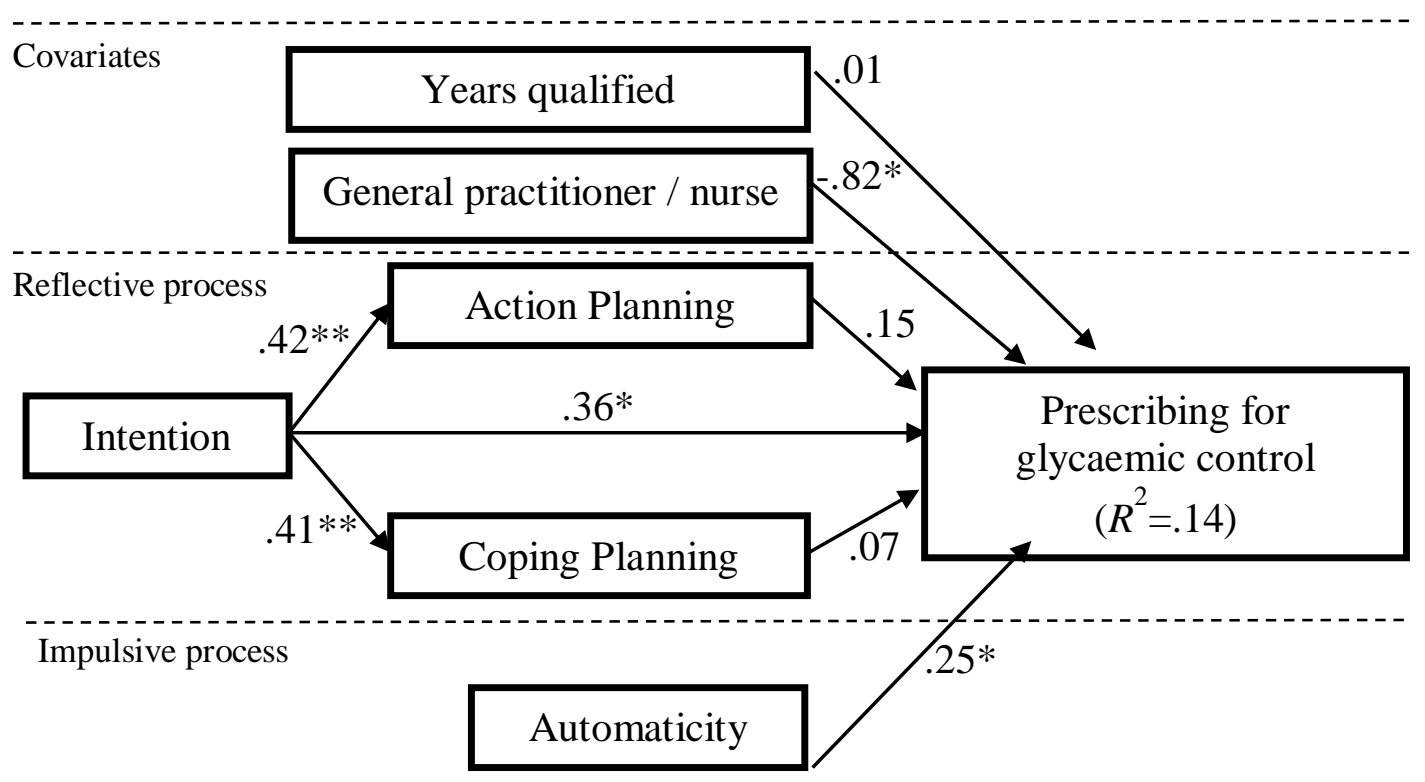

Figure 2. Prescribing for glycemic control: Bootstrapped multiple mediation model simultaneously testing the sequential relationship between intention and behavior via action and coping planning, alongside automaticity, controlling for differences between general practitioners and nurses and years qualified. 


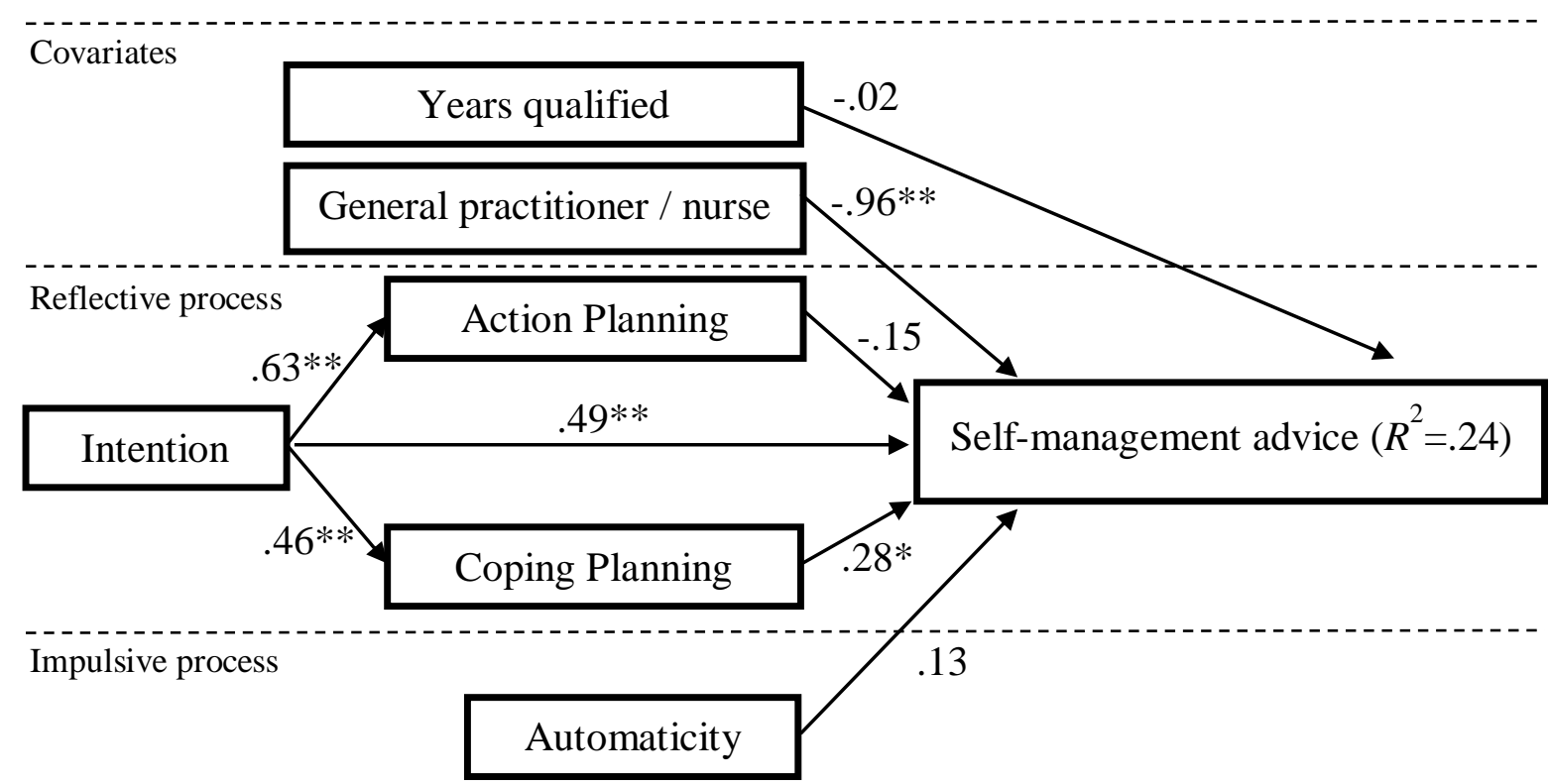

Figure 3. Providing self-management advice: Bootstrapped multiple mediation model simultaneously testing the sequential relationship between intention and behavior via action and coping planning, alongside automaticity, controlling for differences between general practitioners and nurses and years qualified. 


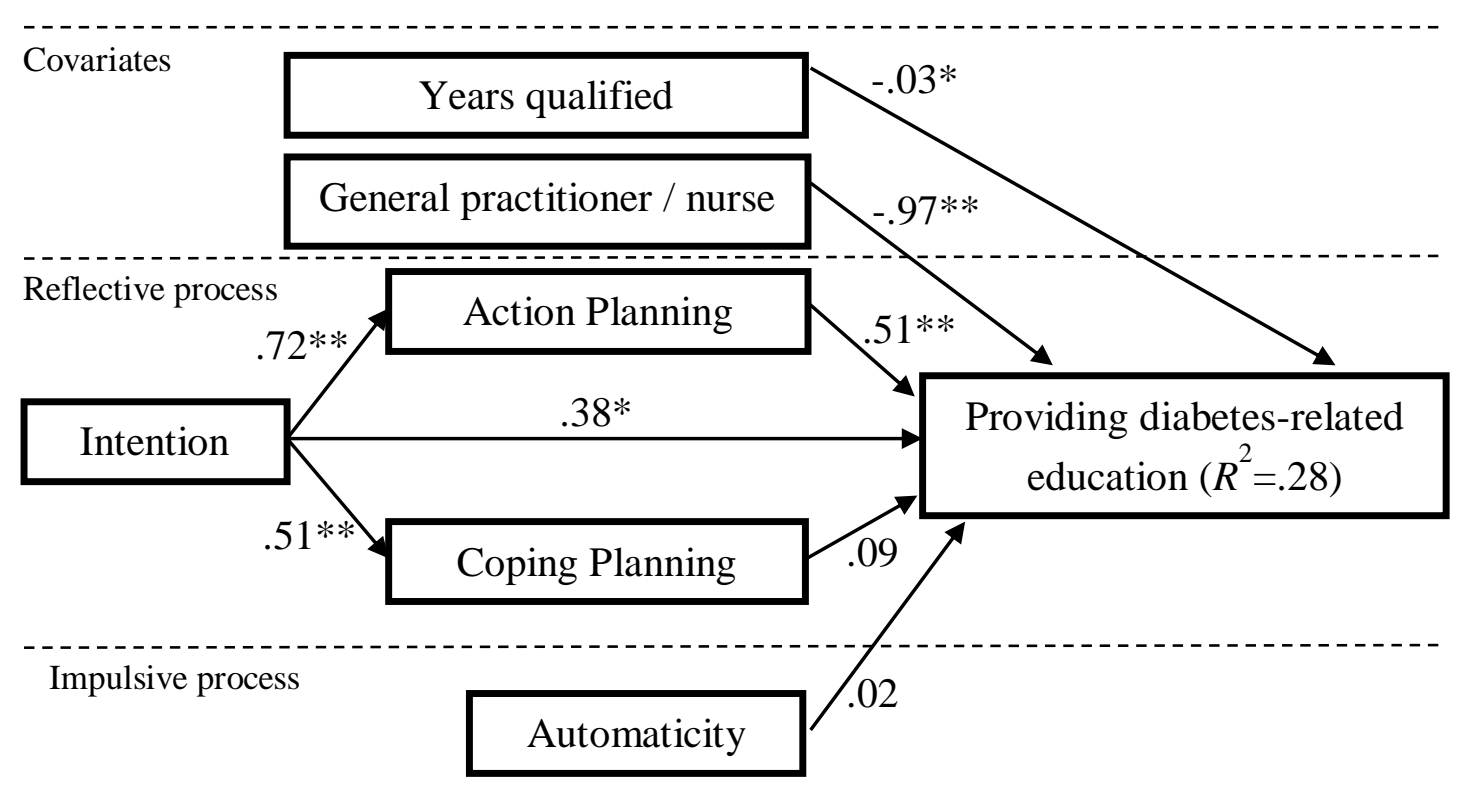

Figure 4. Providing diabetes-related education: Bootstrapped multiple mediation model simultaneously testing the sequential relationship between intention and behavior via action and coping planning, alongside automaticity, controlling for differences between general practitioners and nurses and years qualified. 


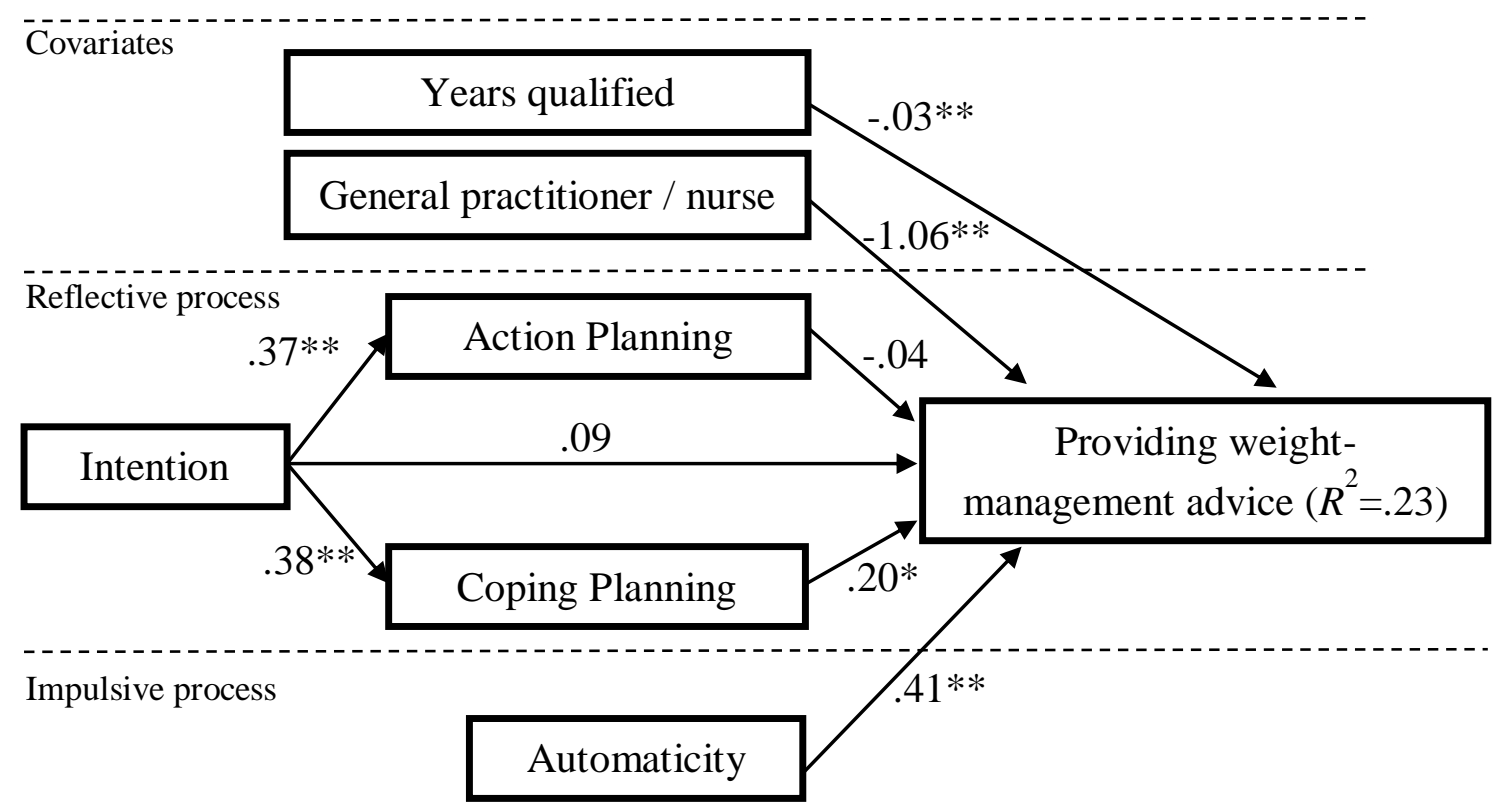

Figure 5. Providing weight management advice: Bootstrapped multiple mediation model simultaneously testing the sequential relationship between intention and behavior via action and coping planning, alongside automaticity, controlling for differences between general practitioners and nurses and years qualified. 


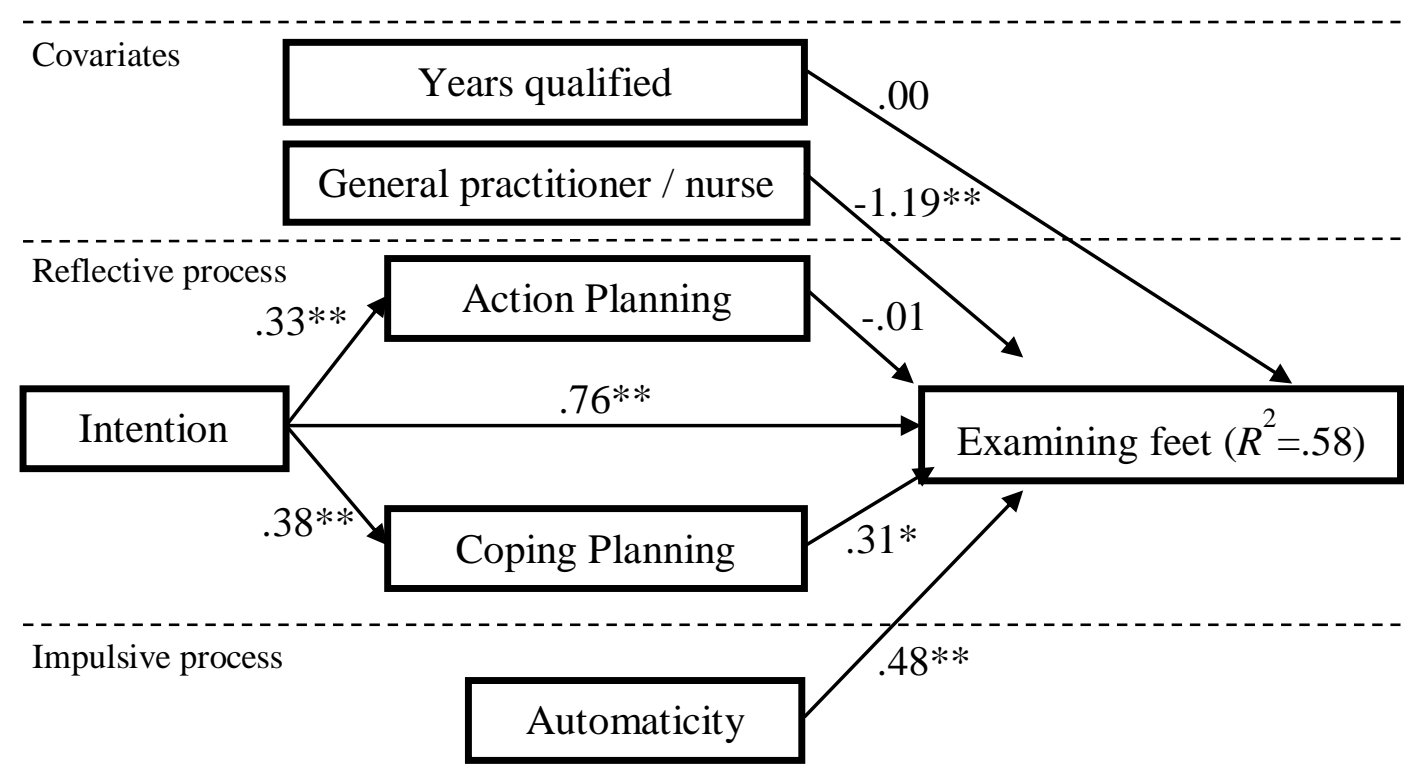

Figure 6. Examining feet: Bootstrapped multiple mediation model simultaneously testing the sequential relationship between intention and behavior via action and coping planning, alongside automaticity, controlling for differences between general practitioners and nurses and years qualified. 